\title{
ON THE MEASURABILITY OF ORBITS IN BOREL ACTIONS
}

\author{
DOUGLAS E. MILLER
}

\begin{abstract}
We replace measure with category in an argument of $\mathbf{G}$. W. Mackey to characterize closed subgroups $H$ of a totally nonmeager, 2nd countable topological group $G$ in terms of the quotient Borel structure $G / H$. As a corollary, we obtain an improved version of a theorem of C. RyllNardzewski on the Borel measurability of orbits in continuous actions by Polish groups.
\end{abstract}

In [9], G. W. Mackey gave an argument using Haar measures to prove the following: Assume $G$ is locally compact topological group. If $H$ is a subgroup such that the space $G / H$, formed by giving the set of (left) $H$-cosets the quotient Borel structure, is countably separated, then $H$ is closed in $G$. We will show that this result can be obtained by an analogous argument using the theory of Baire category. The category version has a wider application and shows that the above statement remains true under the weaker assumption that $G$ is totally nonmeager. In particular, it holds whenever $G$ is topologically complete (in the sense of Čech). As a corollary, we will prove that a well-known theorem of $\mathrm{C}$. Ryll-Nardzewski on the Borel measurability of orbits in continuous actions by Polish (separable, completely metrizable) groups holds for Borel actions as well. We will also show that a recent result of $\mathrm{R}$. Vaught on decompositions of invariant analytic sets has a similar extension to Borel actions.

Each step in our main argument corresponds to an analogous step in Mackey's proof. The reader may find it interesting to compare our development with the proof of Theorem 7.2 in Mackey [9].

Recall (cf. [2, IX, Exercise 5-27]) that a topological group $G$ which is not meager (first category) is a Baire space (i.e. no nonempty open subset of $G$ is meager in $G$ ). $G$ is totally nonmeager if no nonempty closed subset of $G$ is meager in itself. Every topologically complete space, in particular every locally compact space and every completely metrizable space, is totally nonmeager (cf. [7, III, §8]). $B \subseteq G$ is strictly Baire (or has the restricted Baire property)

Presented to the Society, October 25, 1975; received by the editors May 24, 1976 and, in revised form, August 2, 1976.

AMS (MOS) subject classifications (1970). Primary 54H15, 22A05; Secondary 22D05, 54E45, 54E50, 54H05.

Key words and phrases. Transformation group, orbit, Baire space, Polish group, totally nonmeager, Borel action, countably separated Borel space, zero-one law, selector.

1 The author is grateful to L. G. Brown for this remark. 
provided $B \cap C$ is Baire (has the Baire property) in $C$ whenever $C$ is closed in $G$. A function $J: G \rightarrow X$ is strictly Baire measurable if $J^{-1}(B)$ is strictly Baire when $B$ is Borel in $X$. Every Borel set is strictly Baire and every Borel measurable function is strictly Baire measurable.

The identity of a group $G$ will always be denoted by $e$. If $H$ is a subgroup, then $G / H$ is the set of left $H$-cosets and $\pi$ is the projection $g \rightarrow g H$.

The key lemma is a zero-one law for nonmeager groups:

Lemma. Assume that $G$ is a nonmeager topological group and $H$ is a dense subgroup of $G$. Suppose $E \subseteq G$ is Baire and (right) $H$-invariant (i.e. $E H=E$ ). Then $E$ is meager or comeager in $G$.

Proof. This is a special case of the theorem of [3]. A somewhat shorter proof of our lemma (and of (1) $\Rightarrow(4)$ in [3]) can be given using a known technique (cf. [13,3.5]) $-D(E)=\{x: E$ is not meager at $x\}$ is closed, $H$ invariant, and congruent to $E$. Since $H$ is dense, either $D(E)=G$ or $D(E)=\varnothing$.

Theorem 1. Assume that $G$ is a totally nonmeager topological group. Suppose $H$ is a subgroup of $G$ and $\left\{E_{i}: i \in \omega\right\}$ is a collection of $H$-invariant, strictly Baire sets which separates $H$-cosets (i.e. for each $\left.g \in G, g H=\cap\left\{E_{i}: g \in E_{i}\right\}\right)$. Then $H$ is closed in $G$.

Proof. Here and below, let $\bar{H}$ be the closure of $H$ in $G$. By replacing $G$ by $\bar{H}$ and each $E_{i}$ by $E_{i} \cap \bar{H}$, we may assume that $H$ is dense in $G$. We must show $H=G$.

It follows from the lemma that each $E_{i}$ is meager or comeager. Since $H=\bigcap\left\{E_{i}: e \in E_{i}\right\}, H$ is Baire, and it follows from a well-known theorem of Banach, Kuratowski, and Pettis that $H=G$ or $H$ is meager (cf. [8, $\S 13$, XII]). Assume for contradiction that $H$ is meager. Let $g \in G$, then $g H$ is meager. Since $g H=\cap\left\{E_{i}: g \in E_{i}\right\}$ and comeager sets are closed under countable intersections, there is a meager $E_{i}$ which contains $g$. Thus, $G$ $\subseteq \cup\left\{E_{i}: E_{i}\right.$ is meager $\}$ and $G$ is meager. This contradiction shows that $H=G$ as required.

An action of a group $G$ on a set $X$ is a function $J: G \times X \rightarrow X$ such that the map $g \rightarrow J^{g}$ is a homomorphism on $G$ to the group of permutations of $X$. $J^{g}(x)=J(g, x)=J^{x}(g)=g x$.

A metrizable space $X$ is Lusin (absolutely Borel) provided $X$ is the image of a Polish space under a 1-1, continuous map. Every Borel subspace of a Polish space is Lusin and every Lusin space $X$ is Borel in any metrizable extension of $X$ (cf. [8]).

The next theorem combines and extends two known results. The special case " $G$ Polish, $X$ metric, $J^{x}$ continuous" was proved by C. Ryll-Nardzewski in [11]. (A closely related result is in Dixmier [6].) The special case " $G$ 2nd countable, locally compact, $X$ separable metric, $J^{x}$ Borel" follows from results of Mackey (cf. [1, 3.7]). 
Theorem 2. Assume $G$ is a Polish topological group, $X$ is a metric space and $J: G \times X \rightarrow X$ is an action. Suppose $x \in X$ and $J^{x}$ is Borel measurable. Then the stabilizer $H_{x}=\{g: g x=x\}$ is closed in $G$ and the orbit $G x=\{g x: g \in G\}$ is a Lusin space.

Proof. $G x$ is a metrizable image of the Polish space $G$ under the Borel map $J^{x}$, so $G x$ is separable. Let $\left\{B_{i}: i \in \omega\right\}$ be a basis for the relative topology on $G x$. Then $\left\{\left(J^{x}\right)^{-1}\left(B_{i}\right): i \in \omega\right\}$ is a collection of $H_{x}$-invariant Borel sets which separates $H_{x}$-cosets. It follows from Theorem 1 that $H_{x}$ is closed and hence, there is a Borel set $S \subseteq G$ which intersects each $H_{x}$-coset in a singleton (cf. [11] or [6]). $G x$ is the metrizable image of the Lusin space $S$ under the one-one Borel function $J_{\uparrow S}^{x}$. By $\$ 39 \mathrm{~V}(\mathrm{i})$ of [8], $G x$ is Borel in its completion, so $G x$ is Lusin.

REMARK. The fact that a metrizable image of a Polish space $G$ under a Borel map $S$ is separable was apparently first noticed by Calvin Moore, see [5]. The same result with $f$ assumed one-one is a well-known theorem of $\mathrm{A}$. H. Stone, cf. [8, p. 491]. To derive Moore's theorem from Stone's, consider the composition

$$
G \stackrel{1 \times f}{\longrightarrow} \mathcal{G} \stackrel{\pi}{\longrightarrow} f(G)
$$

where $\mathcal{G}=\{(g, f(g)): g \in G\}$ is the graph of $f$, and $\pi$ is the projection map. $\mathcal{G}$ is separable by Stone's theorem, and $f(G)=\pi(\mathcal{G})$ is the continuous image of a separable space, hence separable.

In order to make the connections with Mackey's work more explicit, we next restate Theorem 2 in terms of Borel structures and then give a generalization of Theorem 1 which is analogous to 7.2 of [9].

A Borel space $X$ is a set with a $\sigma$-field $\mathscr{B}$ of distinguished or Borel subsets. $X$ is countably separated if there is a collection $\left\{B_{i}: i \in \omega\right\}$ of members of $\mathscr{B}$ which separates points in $X\left(x=\cap\left\{B_{i}: x \in B_{i}\right\}\right.$ for each $\left.x \in X\right)$. Relative Borel structures and quotient Borel structures are formed in the obvious way (cf. [9] or [1]). A topological space is implicitly given the Borel structure generated by the open sets. A Borel space is standard if it is isomorphic to the Borel structure of a Polish space.

REMARK. If $G$ is a topological group and $H$ is a subgroup, the Borel structure of the quotient topological space $((G / H))$, (which is generated by projections of invariant open sets) is generally weaker than the quotient Borel structure on $G / H$ (generated by projections of invariant Borel sets). If $G$ is Polish and $H$ is closed, then both $G / H$ and $((G / H))$ are countably separated, hence analytic (cf. [9, Corollary to 5.1]). It follows from 4.3 of [9] that the two structures coincide.

THEOREM 2'. Assume that a totally nonmeager topological group $G$ acts on a countably separated Borel space $X$ according to a function $J: G \times X \rightarrow X$. Suppose $x \in X$ and $J^{x}$ is strictly Baire measurable. Then the stabilizer subgroup $H_{x}=\{g: g x=x\}$ is closed. If $G$ is Polish and $J^{x}$ is Borel, then the orbit 
$G x=\{g x: g \in G\} \subseteq X$, given the relative Borel structure, is a standard Borel space and $G x$ is Borel in $X$.

Proof. Let $\left\{B_{i}: i \in \omega\right\}$ be a collection of Borel sets which separates points in $X$. Then $\left\{\left(J^{x}\right)^{-1}\left(B_{i}\right): i \in \omega\right\}$ is a collection of $H_{x}$-invariant, strictly Baire sets which separates $H_{x}$-cosets. It follows from Theorem 1 that $H_{x}$ is closed. As before, if $G$ is Polish, there is a Borel selector $S$ for $G / H_{x}$. If $J^{x}$ is Borel, it follows from 5.1 of Mackey [9] that $G x$ is countably generated. Then it follows from 3.2 of [9] that $G x$ is standard. By Corollary 3 to 3.2 of [9], $G x$ belongs to the $\sigma$-algebra generated by $\left\{B_{i}: i \in \omega\right\}$; a fortiori, $G x$ is Borel in $X$. (In fact, this corollary could be stated, "A subset of a countably separated Borel space which is standard as a Borel subspace is a Borel subset."l)

In [9] Mackey assumes that $G$ has a countable base and proves that $H$ is closed whenever $G$ includes a small (in the sense of measure) $H$-invariant subset $N$ such that $G / H \sim \pi N$ is countably separated. The category version of this argument also requires that $G$ has a countable base. Since it is not required for the application, we have separated it from the main theorem.

THEOREM 3. Assume $G$ is a totally nonmeager topological group with a countable base. Let $H$ be a subgroup of $G$. Then the following are equivalent:

(i) $H$ is closed.

(ii) The quotient Borel structure $G / H$ is countably separated.

(iii) There is an $H$-invariant meager set $N \subseteq G$ and a collection $\left\{E_{i}: i \in \omega\right\}$ of $H$-invariant, strictly Baire sets such that $\left\{E_{i} \sim N: i \in \omega\right\}$ separates $H$-cosets in $G \sim N$.

Proof. (i) $\Rightarrow$ (ii). Let $((G / H))$ be the quotient topological space (i.e. the homogeneous space associated with $H)$. Let $\left\{B_{i}: i \in \omega\right\}$ be a basis for the topology on $G$. If $G$ is closed, then $((G / H))$ is Hausdorff (cf. [2, III, §2.5]), and the set $\left\{\pi\left(B_{i} H\right): i \in \omega\right\}$ witnesses the fact that $G / H$ is countably separated.

(ii) $\Rightarrow$ (iii) trivially.

(iii) $\Rightarrow$ (i). Assume $G, H, N,\left\{E_{i}: i \in \omega\right\}$ satisfy condition (iii). If $H$ is dense in $G$, the argument of Theorem 1 is easily modified to show that $H=G$. Thus, it suffices to prove that we can replace $G$ by $\bar{H}$; i.e. that there is a set $N^{\prime} \subseteq \bar{H}$ and a collection $\left\{D_{i}: i \in \omega\right\}$ of subsets of $\bar{H}$ such that $\bar{H}, H, N^{\prime},\left\{D_{i}: i \in \omega\right\}$ satisfy condition (iii).

$\bar{H}$ is a nonmeager topological group with a countable basis which acts on $G$ by right translation. For $g \in G,\{h \in \bar{H}: g h \in N\}=g^{-1} N \cap H$. Let $N^{\Delta}$ $=\left\{g \in G: g^{-1} N \cap H\right.$ is not meager in $\left.\bar{H}\right\}$. It follows from Theorem 2.4(a) of [4] that $N^{\Delta}$ is meager in $G$. Since $G$ is not meager in itself, $G \sim N^{\Delta} \neq \varnothing$ and there exists $g_{0} \in G$ such that $g_{0}^{-1} N \cap \bar{H}$ is meager in $\bar{H}$. Let $N^{\prime}$ $=g_{0}^{-1} N \cap \bar{H}$, and $D_{i}=g_{0}^{-1} E_{i} \cap \bar{H}$ for each $i \in \omega$. Since the map $g \mapsto g_{0}^{-1} g$ is an automorphism of $G$ and $\bar{H}$ is closed, each $D_{i}$ is strictly Baire in $\bar{H}$. If $h \in \bar{H} \sim N^{\prime}$, then $g_{0} h \in G \sim N$ and $g_{0} h H=\cap\left\{E_{i} \sim N: g_{0} h\right.$ $\left.\in E_{i}\right\}$. It follows that 


$$
h H=\cap\left\{g_{0}^{-1} E_{i} \sim g_{0}^{-1} N: h \in g_{0}^{-1} E_{i}\right\}=\bigcap\left\{D_{i} \sim N^{\prime}: h \in D_{i}\right\} .
$$

Thus, $\left\{D_{i} \sim N^{\prime}: i \in \omega\right\}$ separates cosets in $G \sim N^{\prime}$ as required.

In [13, 2.5 and 2.6], R. L. Vaught obtained a result on orbits which is closely related to that of Ryll-Nardzewski. By exploiting the connection between Vaught's general assumptions and his "product case" we can extend several of his main results about continuous actions to the case of Borel actions. In particular, we improve his general theorem $([13,2.5])$ on the decomposition of invariant analytic sets and (consequently) obtain a second proof that orbits are Borel in any Borel action of a Polish group on a countably separated Borel space.

Let $X, X^{\prime}$ be topological spaces, $G$ a Baire space with a countable weak basis [13], and $J: G \times X \rightarrow X^{\prime}$ a Borel measurable function. For $B \subseteq X^{\prime}$ define $B^{* J}=\{x: g: J(g, x) \in B\}$ is comeager $\}$ as in [13]. A set is analytic if it can be obtained from Borel sets by the operation $(\mathscr{A})$. $C$ is the smallest family including the Borel sets and closed under complementation and the operation $(\mathscr{A})$.

Theorem 4. Assume $G, X, X^{\prime}, J$ satisfy the above conditions.

(a) If $B \subseteq X^{\prime}$ is respectively Borel, analytic, or $\varrho$, then the same is true of $B^{*}$.

(b) Assume additionally that $X=X^{\prime}, G$ is a topological group, and $J$ defines an action of $G$ on $X$. Then every invariant analytic subset of $X$ is a union of $\aleph_{1}$ invariant Borel sets.

Proof. (a) and (b) are proved in [13] under the stronger hypothesis that $J$ is continuous in each variable. We easily reduce our theorem to Vaught's as follows. Fix $B \subseteq X^{\prime}$ and let $A=J^{-1}(B)$. Let $I: G \times X \rightarrow G \times X$ be the identity map and notice that for every $x,\{g: J(g, x) \in B\}=\{g: I(g, x)$ $\in A\}$. Thus, $B^{* J}=A^{* I}$. Since $I$ is continuous and each of the collection of Borel, analytic, and $C$ sets is closed under inverse Borel images, our conclusion is immediate from the corresponding results in [13]. In verifying (b), note that the proof $[13,2.1(\mathrm{a})]$ establishes the invariance of $B^{*}$ in our more general setting.

Remarks. 1. Assume $G$ is a Polish group, $X$ a countably separated Borel space, $J: G \times X \rightarrow X$ a Borel action. Then the Borel structure on $X$ is compatible with a metric topology; hence, orbits are analytic (and minimal invariant). It follows directly from 4.1(b) that orbits are Borel. (Compare [13, 2.6].) Using our Theorem $2^{\prime}$, Vaught has recently obtained positive results on the measurability of orbits in certain cases where $X$ is not countably separated. He has also discovered two arguments extending our 4(a) to the case where $X$ and $X^{\prime}$ are arbitrary Borel spaces.

2. Assuming that $G$ is a locally compact, 2 nd countable topological group acting by a Borel map $J: G \times X \rightarrow X$ on an analytic Borel space $X$, V. S. Varadarajan (cf. [12, 3.2 and 3.5]) obtained the fact that each stabilizer $H_{x}$ is closed as a consequence of a normal form theorem; viz.: In this case there is a 
metric on $X$, which is compatible with the Borel structure and which makes $J$ a continuous map. We do not know whether an analogous result holds assuming only that $G$ is Polish. Note however, that every quotient of a metrizable group by a closed subgroup is separable and regular, hence metrizable (cf. [10]). Therefore, the normal form result follows from Theorem 2 if we add the assumption that the action is transitive (i.e. $X$ is a single orbit).

\section{REFERENCES}

1. L. Auslander and C. C. Moore, Unitary representations of solvable Lie groups, Mem. Amer. Math. Soc. No. 62 (1966). MR 34 \#7723.

2. N. Bourbaki, Elements of mathematics. General Topology. Parts 1, 2, Hermann, Paris; Addison-Wesley, Reading, Mass., 1966. MR 34 \# 5044a, b; erratum, 40, p. 1704.

3. M. Bhaskara Rao and K. P. S. Bhaskara Rao, A category analogue of the Hewitt-Savage zeroone law, Proc. Amer. Math. Soc. 44 (1974), 497-499. MR 49 \#9823.

4. J. P. Burgess and D. E. Miller, Remarks on invariant descriptive set theory, Fund. Math. 99 (1975), 53-75.

5. D. L. Cohn, Measurable choice of limit points and the existence of separable and measurable processes, Z. Wahrscheinlichkeitstheorie und Verw. Gebiete 22 (1972), 161-165. MR 46 \#4574.

6. J. Dixmier, Dual et quasi-dual d'une algèbre de Banach involutive, Trans. Amer. Math. Soc. 104 (1962), 278-283. MR 25 \# 3384.

7. R. Engelking, Outline of general topology, PWN, Warsaw, 1965; English transl., NorthHolland, Amsterdam; PWN, Warsaw; Interscience, New York, 1968. MR 36 \#4508; 37 \# 5836.

8. K. Kuratowski, Topology, Vol. I, Vol. II, PWN, Warsaw; Academic Press, New York, 1966, 1968. MR 36 \#839, 840; 41 \#4467, 4468.

9. G. W. Mackey, Borel structure in groups and their duals, Trans. Amer. Math. Soc. 85 (1957), 134-165. MR 19, 752.

10. L. S. Pontryagin, Topological groups, 2nd ed., GITTL, Moscow, 1954; English transl., Gordon and Breach, New York, 1966. MR 34 \# 1439.

11. C. Ryll-Nardzewski, On Borel measurability of orbits, Fund. Math. 56 (1964), 129-130. MR 30 \# 3028.

12. V. S. Varadarajan, Groups of automorphisms of Borel spaces, Trans. Amer. Math. Soc. 109 (1963), 191-220. MR 28 \#3139.

13. R. L. Vaught, Invariant sets in topology and logic, Fund. Math. 82 (1974/75), 269-294. MR 51 \#167.

Department of Mathematics, Yale University, New Haven, Connecticut 06520 